Image processing and control of a programmable spatial light modulator

A. Awwal, R. Leach, G. Brunton, E. Tse, J. Matone, J. Heebner

February 10, 2011

SPIE Photonics West

San Francisco, CA, United States

January 22, 2011 through January 27, 2011 
This document was prepared as an account of work sponsored by an agency of the United States government. Neither the United States government nor Lawrence Livermore National Security, LLC, nor any of their employees makes any warranty, expressed or implied, or assumes any legal liability or responsibility for the accuracy, completeness, or usefulness of any information, apparatus, product, or process disclosed, or represents that its use would not infringe privately owned rights. Reference herein to any specific commercial product, process, or service by trade name, trademark, manufacturer, or otherwise does not necessarily constitute or imply its endorsement, recommendation, or favoring by the United States government or Lawrence Livermore National Security, LLC. The views and opinions of authors expressed herein do not necessarily state or reflect those of the United States government or Lawrence Livermore National Security, LLC, and shall not be used for advertising or product endorsement purposes. 


\title{
Image processing and control of a programmable spatial light modulator
}

\author{
Abdul A. S. Awwal, Richard Leach, Gordon Brunton, Eddy Tse, JoAnn Matone and John Heebner \\ Integrated Computer Control System, National Ignition Facility \\ Laser Science Engineering and Operations \\ Lawrence Livermore National Laboratory, Livermore, CA. 94551 \\ E-mail: awwal1@,llnl.gov
}

\begin{abstract}
The heart of the National Ignition Facility is a megajoule-class laser system consisting of 192 beams used to drive inertial confinement fusion reactions. A recently installed system of programmable, liquid-crystal-based spatial light modulators adds the capability of arbitrarily shaping the spatial beam profiles in order to enhance operational flexibility. Its primary intended use is for introducing "blocker" obscurations to shadow isolated flaws on downstream optical elements. To optimize the system, both the position and shape of the obscurations must be carefully verified prior to high-fluence operations. An automatic alignment algorithm is used to perform detection and estimation of the imposed blocker centroid positions compared to their intended locations. Furthermore, in order to minimize the spatially-varying nonlinear response of the device, a calibration of the local magnification is performed at multiple sub-image locations. In this paper, we describe the control and associated image processing of this device that helps to enhance the performance of the overall system.
\end{abstract}

Key word: pattern recognition, line detection, laser alignment, spatial light modulator

\section{INTRODUCTION}

The National Ignition Facility (NIF), a 192-beam, 1.8 megajoule, 500-terawatt, ultraviolet, laser system is used for inertial confinement fusion research at the Lawrence Livermore National Laboratory [1]. The integrated computer control system is utilized to manage key functions of NIF laser such as specifying pulse shape, alignment, amplification and beam control [2-4] in order to produce proper timing, high-energy density and pressure leading to a controlled fusion reaction. Various subsystems under computer control perform alignment, optics inspection and shot planning, etc. An automatic alignment (AA) system analyzes beam images to determine the location of the laser in order to guide the control system to perform optical alignment. The AA system is equipped with image processing modules capable of processing various beam images to extract pertinent information. The optics inspection (OI) system is used to identify flaws of different sizes on various optical elements in the beam path and track them over time.

One of the challenges of operating NIF is to maintain the high quality of the optical elements that are subjected to repetitive laser shots. Exposure to high-fluence laser light can cause some optical flaws to deteriorate over time and impact performance. Recently a programmable spatial shaper (PSS) based on liquid-crystal spatial light modulators (SLM) was designed and implemented within the NIF laser for arbitrarily shaping obscurations that act to reduce laser irradiation around selected flaw sites [5]. The OI system supplies the flaw site information, while the PSS system provides "blockers" that cast a shadow in the beam effectively reducing flaw irradiation exposure.

To control the operation of the PSS module, an integrated computer control subsystem was developed. The control system is in charge of creating the blockers of various sizes as specified by OI and verifying that they are correctly 
placed at the specified locations. An image of the beam imprinted with the specified blockers is passed to the image processing module, which verifies that the PSS blocker positions and characteristics match the desired specifications. A secondary challenge is to characterize the SLM in terms of coordinate mapping and imposed blocker shape distortion. In this paper, we describe the control and associated image processing of the PSS subsystem. Next we briefly describe the optical subsystem [5].

\section{PSS: OPTICAL SYSTEM}

In order to control the irradiance of flaws on high-fluence optics, shadows created by PSS blockers are introduced in the low-fluence region of the beam path, known as the pre-amplifier module or PAM. Prior to development of the PSS, static blockers fabricated from chrome masks were installed in the PAM. However, such an approach is inflexible and incapable of meeting the demands of real-time operation. To overcome this problem, a programmable method of creating blockers under computer control was sought. Commercially available pixelated SLMs were attractive but not suitable because of problems with diffraction, wavefront and spectral distortions. An optically-addressable transmissive light valve technology was selected. As shown in Figure 1, the programmable blockers were introduced in the PAM with a goal to project the blockers downstream to shadow flaws in the high-fluence region of the final optics assembly.

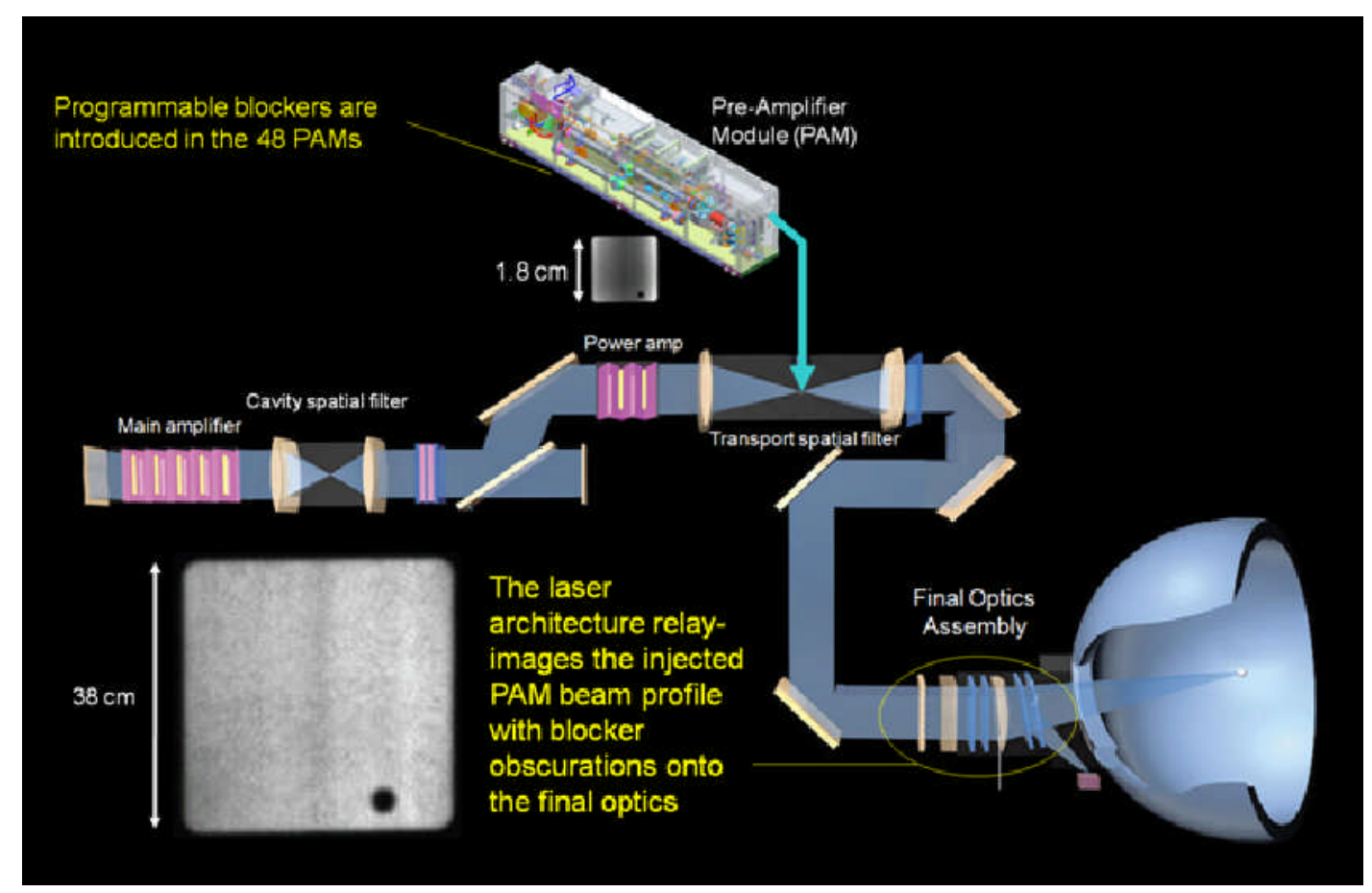

Figure 1. A diagram showing the relative location of the PSS unit (in the PAM) compared to the overall beam path, the final optics assembly and the target chamber.

The beam shaping is accomplished in a two step process. In the first step, the desired bitmapped blocker image is imprinted onto an incoherent beam, using a standard 1920 × 1080 pixel Liquid Crystal on Silicon (LCoS) modulator. The incoherent beam writes the information on the write-side of an optically addressed light valve (OALV), which consists of a large, single pixel twisted nematic liquid crystal cell in series with a layer of photoconductive Bismuth Silicon Oxide (BSO). The incident light pattern alters the spatial voltage around a fixed bias to the liquid crystal layer, which in turn modulates the polarization of the $1053 \mathrm{~nm}$ coherent laser beam. A downstream polarizer converts the polarization modulation into amplitude modulation. Consequently, apodized patterns free from spurious pixelization artifacts are created by this second stage, which imprints the coherent main laser beam with the desired blocker pattern. 


\section{CONTROL OF PSS}

An improperly shaped blocker pattern reduces system performance. Both the position and shape of the obscurations must be carefully verified prior to high-fluence operations. Furthermore, in order to minimize the spatially varying nonlinear response of the device, a calibration of the local magnification is performed at multiple sub-image locations. Therefore, the operation of PSS is performed in a sequence of steps. First, a calibration is performed in order to ensure the spatial matching of the position and size of the obscuration with respect to the deployed beam blockers. In the second step, the mapping information is used to deploy the actual blockers to block the flaw sites. In both cases, automatic alignment algorithms are used to perform detection and estimation of the imposed blocker centroid positions. Thirdly, verification is performed to ensure blockers are properly deployed with respect to position, size, and the contrast.

\subsection{PSS Calibration}

The PSS system calibration is critical to the accurate placement and scaling of blocker obscurations. All blocker locations specified within the system are specified relative to the input sensor package (ISP) CCD image plane (640x480 pixels) as shown in Figure 2. PSS System calibration provides the PSS controller with the factors necessary to translate between the ISP CCD pixel locations and the LCoS SLM pixel locations where the blockers are rendered. Calibration consists of two main phases, commissioning and operation.

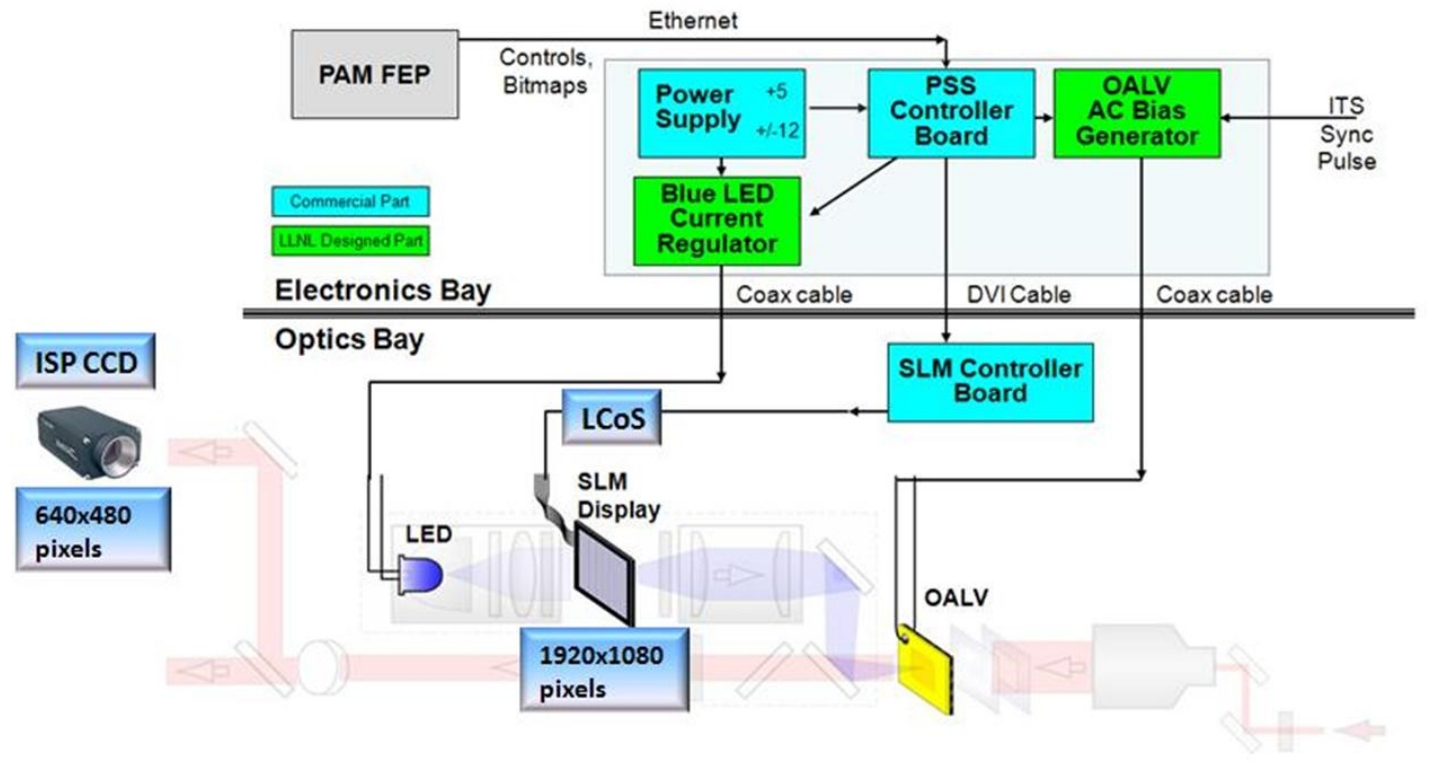

Figure 2. PSS Control Point Overview. The "PAM FEP" at top is the pre-amplifier module front-end processor.

\subsubsection{Commissioning Calibration}

The PSS system control uses two image planes - the LCoS SLM display (1920x1080 pixels) on which the blocker obscurations are rendered, and the ISP CCD camera (640x480 pixels) that is used to verify that blocker obscurations are 
correctly located and have the requested diameter. These two image planes have independent resolutions and offsets. Commissioning calibration grossly calibrates the deployment (LCoS) image plane to the verification (ISP) image plane by measuring both the $\mathrm{x} / \mathrm{y}$ offset and the scaling factor required to transform between the two planes. These calibration factors are unique for each PSS system and are generally only measured and recorded during the installation of each PSS system or when maintenance is performed on the system. The accuracy of these gross factors is not particularly critical as any minor variances are removed by the autonomous fine operational calibration performed on each blocker deployment activity as part of every NIF shot cycle.

\subsubsection{Operational Calibration}

As the NIF optical beamlines are aligned on every shot cycle, small variances in PSS alignment can occur from shot to shot. To address these variances the PSS system requires two operational calibrations during each shot cycle to ensure the accuracy of blocker deployments, and thus minimizing the laser energy exposure on the corresponding final optic flaw locations.

The commissioning calibration activity provides the offset and scale factors necessary to place blockers in approximate location required for a shot. The first operational calibration activity involves refining these gross factors to allow more precise placement of blockers within the beam aperture. Due to several optical and system factors, spatial distortions exist across the entire beam aperture. To compensate, the first phase of operational calibration is the deployment of a 5by-5 grid of blockers to measure these distortions at known discrete locations across the beam aperture. Using image processing routines to accurately locate the centroid and diameter of these blockers, the calibration routine autonomously adjusts the deployed blocker grid locations and diameters until both are within tolerance of the requested settings. This grid blocker is shown in Figure 3.

As all blocker locations are specified in relation to a static fiducial reference located at center of the square cutout on the left alignment wing mask, the final operational calibration uses image processing to calculate the offset from the image origin to the center of the fiducial.

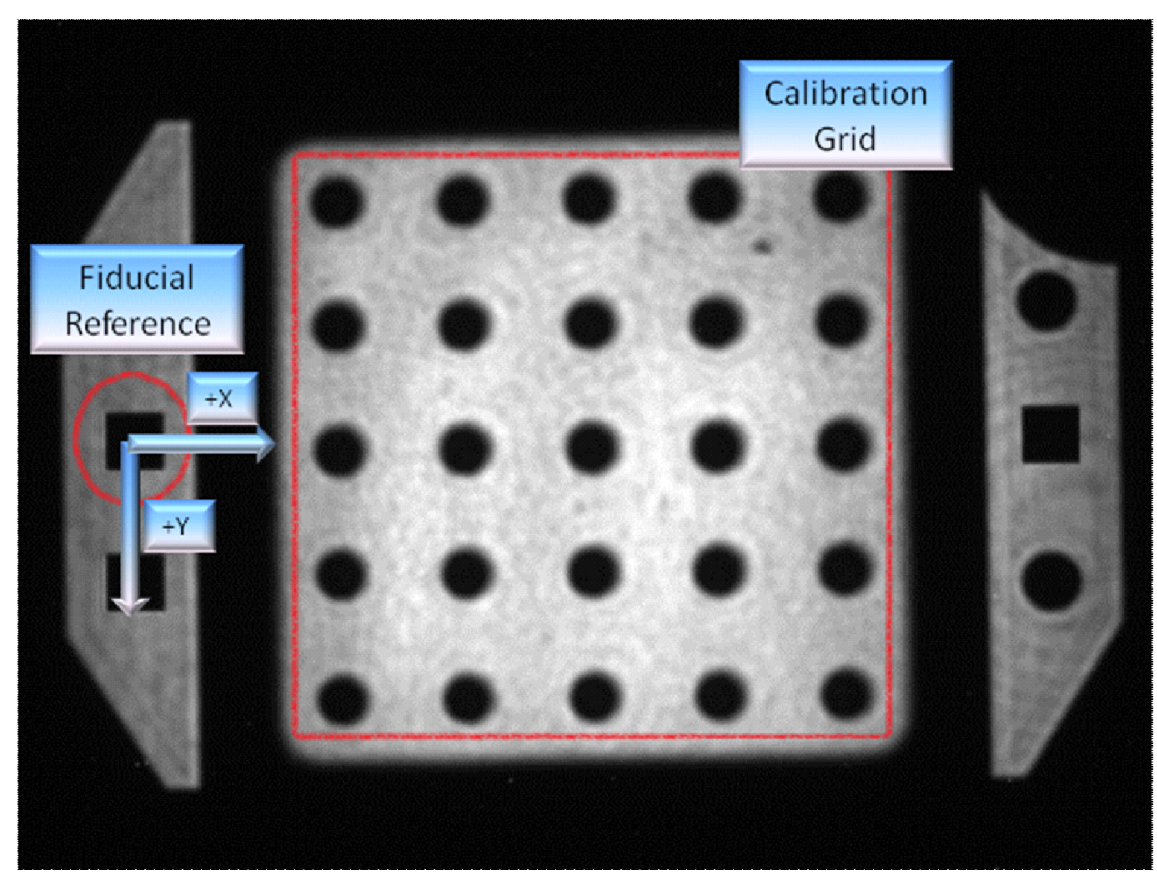

Figure 3. PSS Operational Calibration Components. 
Once commissioning and operational calibration are complete, the PSS controller stores all calibration factors that will then be used for the deployment of the blockers required for a specific shot. To recap, the factors recorded during the calibration activities are:

a) ISP to LCoS offset factors ( $x, y)$

b) ISP to LCoS scale factor

c) Fine offset and diameter correction factors for each calibration grid location

d) Fiducial reference offset.

Calibration is performed on the PSS system as a predecessor to the blocker deployment, rather than incorporating the calibration activity into the blocker deployment activity, due to issues encountered when attempting to perform 'on-thefly' calibration of overlapping blockers and blockers that may intersect the edge of the beam aperture. Performing the calibration up front has proven to increase the robustness of the PSS system as it is unaffected by arbitrary shot blocker locations (which due to the optics inspection and analysis processing can include locations almost entirely outside the beam aperture).

\subsection{Blocker Deployment}

PSS blockers are deployed and verified for each NIF shot cycle. The quantity and location of blockers deployed on each beam depends on two factors: 1) the location and severity of the optic flaw that exists in the downstream beamline optic, and 2) the energy requirements of the shot experiment. Optic flaws are analyzed on a periodic basis and recorded with the system configuration. Each flaw is characterized with details specifying the energy level to which the flaw can be exposed before it could result in degradation. During the shot experiment setup calculations, each optic flaw is evaluated against the shot energy level to determine whether it is required to be blocked. These calculations complete the specification of the set of blockers for each of the NIF beamlines participating in the shot. Using automated transformation modeling the optic flaw location is transformed into the ISP CCD location for a blocker in order to minimize the fluence at the downstream flaw site.

During the implementation phase of a NIF shot cycle, the required set of shot blockers is deployed into the PSS system. The deployment uses the offset and scale factors recorded during the calibration phase activities. As optic flaws can be in several forms there are no restrictions imposed on the prescribed placement of the deployed blockers (i.e. overlaps, edge cases).

As each blocker is specified in the ISP CCD pixel coordinates, they are required to be transformed into the LCoS coordinates prior to rendering. Transformation between the two coordinate systems necessitates adjustment and scaling of the requested blocker by the calibration offsets previously calculated.

\subsubsection{Offset Adjustment}

Due to spatial beam aperture variances, each blocker is adjusted using the grid offset and diameter factors measured during the calibration phase. For each shot blocker location, a weighted average of the closest four calibration grid blocker offsets and diameter correction factors are employed. The weighted $\mathrm{x}$ coordinate offset, $\mathrm{U}_{\mathrm{x}}$ is calculated as follows:

$U_{x}=\frac{\left(\left(W o A \times a_{x}\right)+\left(W o B \times b_{x}\right)+\left(W o C \times c_{x}\right)+\left(W o D \times d_{x}\right)\right)}{(W o A+W o B+W o C+W o C)}$

where,

$W o A=\frac{A+B+C+D}{4 \times A}=$ weight of closest point

$W o B=\frac{A+B+C+D}{4 \times B}=$ weight of $2^{\text {nd }}$ closest point 
$W o C=\frac{A+B+C+D}{4 \times C}=$ weight of $3^{\text {rd }}$ closest point

$W o D=\frac{A+B+C+D}{4 \times D}=$ weight of $4^{\text {th }}$ closest point

and

$A, B, C, D=$ distance from blocker center to the $2^{\text {nd }}, 3^{\text {rd }}, 4^{\text {th }}$ closest Calibrated point

$a, b, c, d=$ calibrated offset for Calibration point corresponding to $A, B, C, D$

$\mathrm{a}_{\mathrm{x}}, \mathrm{b}_{\mathrm{x}}, \mathrm{c}_{\mathrm{x}}, \mathrm{d}_{\mathrm{x}}$ calibrated $\mathrm{x}$ coordinate offset for Calibration point corresponding to $A, B, C$, and $D$.

$\mathrm{U}_{\mathrm{y}}$ and $\mathrm{U}_{\mathrm{d}}$ are calculated in a similar manner for the y coordinate offset and diameter correction, respectively.

\subsubsection{ISP to LCoS Coordinate Transformation}

Using the calibration factors and offset adjustment calculations each PSS blocker is rendered on the LCoS SLM at the centroid pixel locations calculated using the following formulas for the LCos blocker $\mathrm{x}$ and y coordinates $\left(\mathrm{L}_{\mathrm{x}}, \mathrm{L}_{\mathrm{y}}\right)$ and diameter $\left(\mathrm{L}_{\mathrm{d}}\right)$ :

$$
\begin{aligned}
& L_{x}=\left(O_{x}+F_{x}+B_{x}+U_{x}\right) \times S \\
& L_{y}=\left(O_{y}+F_{y}+B_{y}+U_{y}\right) \times S \\
& L_{d}=\left(B_{d}+U_{d}\right) \times S
\end{aligned}
$$

where,

$\mathrm{O}_{\mathrm{x}}=$ ISP to LCoS $\mathrm{x}$ offset factor

$\mathrm{O}_{\mathrm{y}}=$ ISP to LCoS y offset factor

$\mathrm{S}=$ ISP to LCoS scale factor

$\mathrm{F}_{\mathrm{x}}=$ Fiducial reference $\mathrm{x}$ offset

$\mathrm{F}_{\mathrm{y}}=$ Fiducial reference $\mathrm{y}$ offset

$\mathrm{B}_{\mathrm{x}}=$ Requested shot blocker ISP $\mathrm{x}$ coordinate

$\mathrm{B}_{\mathrm{y}}=$ Requested shot blocker ISP y coordinate

$\mathrm{B}_{\mathrm{d}}=$ Requested shot blocker ISP diameter

$\mathrm{U}_{\mathrm{x}}=$ Calculated weighted $\mathrm{x}$ coordinate offset

$\mathrm{U}_{\mathrm{y}}=$ Calculated weighted y coordinate offset

$\mathrm{U}_{\mathrm{d}}=$ Calculated weighted diameter correction.

\subsubsection{Apodization}

As optic performance can degrade in beam aperture areas with rapid fluence gradients (i.e. the blocker edges), apodization or blurring is required to be applied to the edge of each blocker to smooth the fluence transitions. When rendering each blocker on the $\mathrm{LCoS}$, the grey scale value of the blocker pixels, $P_{d}$, are adjusted based on the following apodization function. 
$P_{d}=\frac{P_{i}}{2} \times \operatorname{abs}\left(\operatorname{erf}\left(\frac{\sqrt{\left(x_{p}-x_{b}\right)^{2}-\left(y_{p}-y_{b}\right)^{2}}-\frac{d_{b}}{2}}{w}\right)+1\right)$

where,

$P_{i}=$ Blocker pixel grayscale [0..255]

erf $=$ standard gauss error function

$x_{p}=\mathrm{x}$ coordinate of pixel being evaluated

$y_{p}=\mathrm{y}$ coordinate of pixel being evaluated

$x_{b}=\mathrm{x}$ coordinate of blocker

$y_{b}=\mathrm{y}$ coordinate of blocker

$d_{b}=$ full width half depth diameter of blocker

$w=$ edge apodization extent (pixels).

\subsection{Beam Blockers}

Depending on the position of a flaw on an optic, two main types of blockers may be deployed. Isolated flaws could be mitigated by a blocker of a single radius at that particular location. When the size of the flaw is bigger or extends to a certain length, multiple overlapping blockers may be deployed. Figure 4 shows such an overlapped blocker pattern on the right. When there is a need to trim the geometry of the beam perimeter, a line blocker may be used. Line blockers are defined by a straight line at certain angle and distance from the center of the beam as shown in Figure 5 .

\subsubsection{Circle blockers}

A circle blocker may be deployed in two instances - one for calibration and the other for the actual blocking operation. In calibration, a square pattern of $n \times n$ blockers is deployed as previously shown in Figure 3. The position and size of the calibration blockers are determined by the AA algorithm. Two examples of designed blocker patterns are shown in Figure 4.
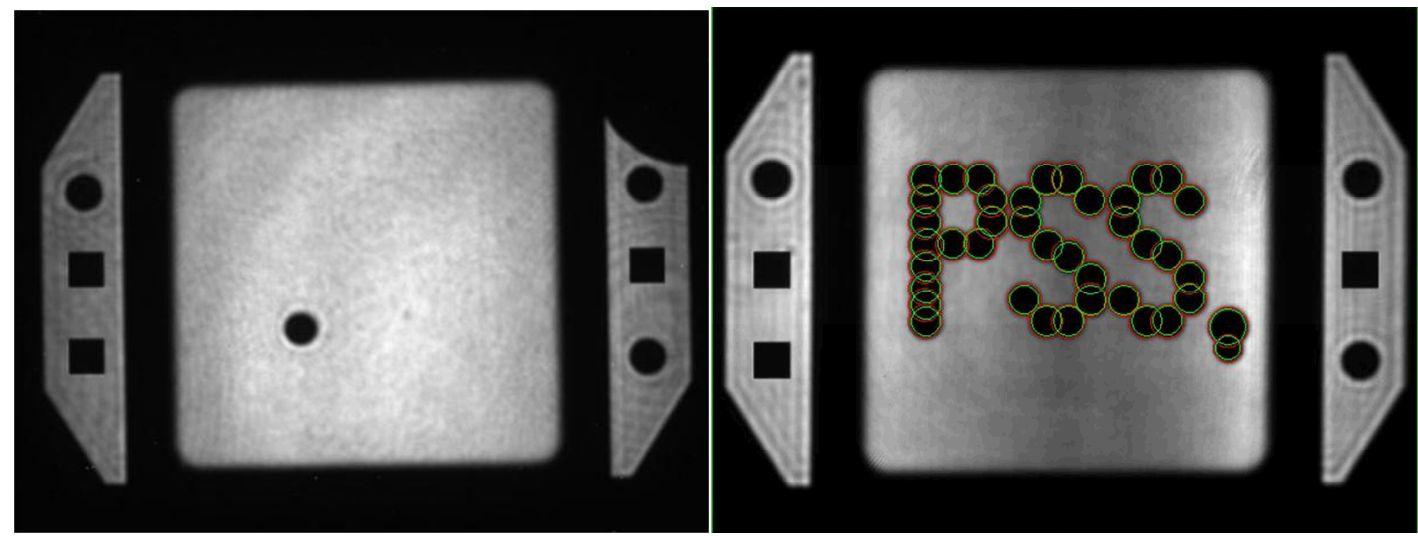

Figure 4. Single circle blocker (left) and multiple detected locations (right) with detected locations in green and desired locations in red. 


\subsubsection{Line blockers}

Any number of line blockers can be used and each blocker can be positioned in any configuration. When line blockers are deployed, a verification process is also performed. A set of equations for the nominal value of the blocker edges is used to create bounding boxes in the image as illustrated in Figure 5. Within the bounding box, the best edge is calculated to provide a measured estimate of the blocker edge. The estimate is compared with the nominal input value, and if they match within the requirement specifications, the blocker is reported as normal. This process is repeated until all known blockers have been estimated and verified as normal.
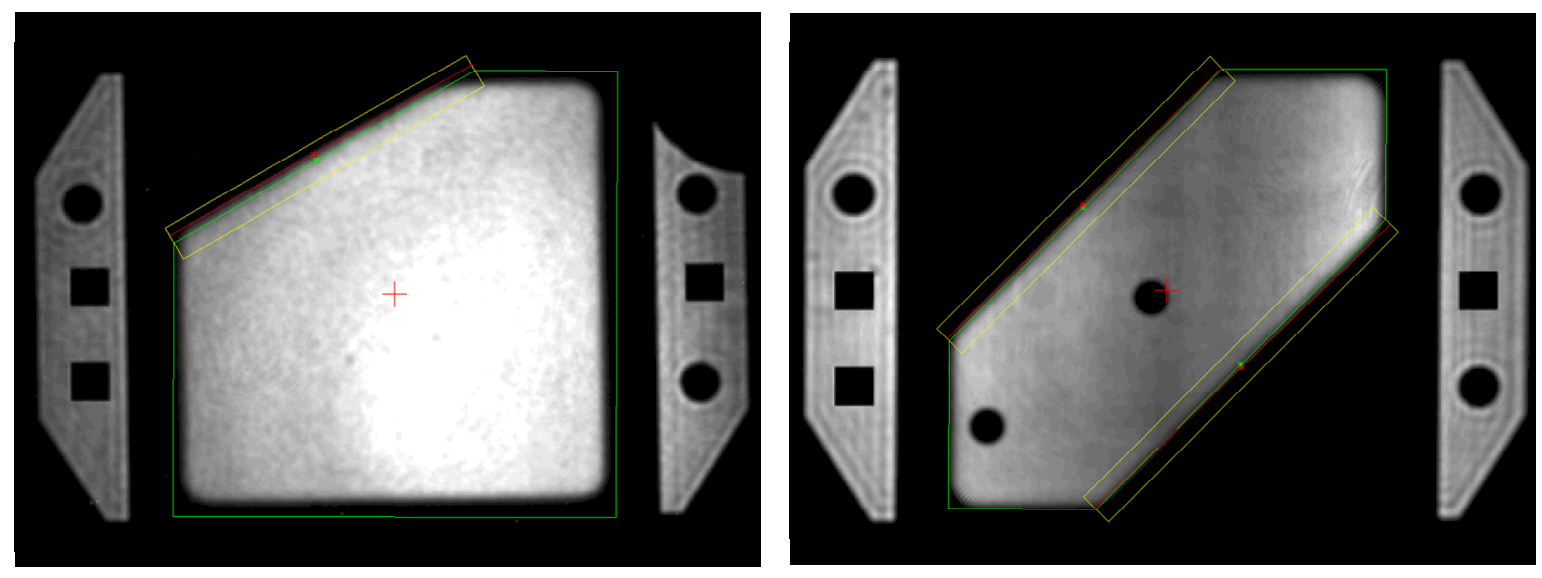

Figure 5. Line blockers with detected locations in green and desired locations in red.

\subsection{Validation}

For a blocker to be verified, three blocker properties are confirmed: location, size and opacity. These properties are considered acceptable if the deviation of the detected parameters is within a certain range. If the location is more than three pixels away from the specified position, then it is considered to have failed. If the size of the detected blocker is bigger than specified then it is acceptable; if smaller, it is not accepted. The darkness is accepted if it is less than a certain threshold.

\section{IMAGE PROCESSING}

Once the blockers are placed on the SLM, the pass-through SLM image is captured by a camera and sent to the AA image processing unit. Depending on the three different types of blockers three different functionalities are accomplished by three different AA image processing units: calibration, circle-blockers and line-blockers units. For the circle blockers, matched filtering is used to detect their position.

\subsection{Matched filtering}

Matched filtering (MF) [6,7] has been successfully utilized to determine the location of beam fiducials with distinct shapes $[8,9]$. One of the chief advantages of the technique is that MF can be applied to the analog domain image without performing extensive preprocessing. Matched filtering techniques rely on the fact that the position of the highest correlation peak is an indicator of the position of the fiducial. 
The matched filtering algorithm utilized here is performed in the frequency domain using a filter defined by

$$
H_{C M F}\left(U_{x}, U_{y}\right)=F^{*}\left(U_{x}, U_{y}\right)=\left|F\left(U_{x}, U_{y}\right)\right| \exp \left(-j \Phi\left(U_{x}, U_{y}\right)\right)
$$

where, $U_{x}, U_{y}$, are the spatial frequency domain variables and $\Phi$ is the phase of the Fourier transform of the to-besearched pattern function $f(\mathrm{x}, \mathrm{y})$ is denoted by:

$$
F\left(U_{x}, U_{y}\right)=\left|F\left(U_{x}, U_{y}\right)\right| \exp \left(j \Phi\left(U_{x}, U_{y}\right)\right)
$$

The product of Equations (4) and (5) produces the correlation in the Fourier domain. Then the inverse Fourier transform of the product produces the auto- or cross-correlation. When (5) is replaced by the Fourier transform of the scene, crosscorrelation between the template and the image results. The performance of the matched filter can be further enhanced by extracting the edge of the image and using the edge of the to-be-detected features as the filter. This has an equivalent effect of high-pass filtering the correlation output, thus increasing the sharpness of the peaks [9]. The position of the object can be found from the position of the cross correlation peak, autocorrelation peak, and the position of the templates.

\subsection{Detecting the circles for blockers and calibration}

For calibration blockers, such as shown in Figure 3, the approximate blocker position is determined by centroiding and later refined by matched filtering. The first step of centroiding is to segment each blocker through a binarization of the analog image. However, since the PAM image is modulated by an additional intensity mask, it imposes a quadratic gradient on the image; which makes the binarization difficult. Therefore a reference image - a PAM image without any blockers - is used to flatten the image intensity. After the binarization, an approximate centroid location is calculated for each blocker location. Starting with these locations and approximate sizes, a matched filter is used to refine the radius for each circle on the edge-detected binary sub-image. For calibration images, the various steps for detecting the location and size of each calibration blocker are depicted in the block diagram of Figure 6.

For the circle blockers, as shown in Figure 4, the supplied positions of blockers are used as input. Note that centroiding will not be very effective, since many blockers are usually overlapped. After flattening and edge-detection, a sub-image is formed by segmenting the image around the input blocker position. The matched filter is used on individual subimages to refine the radius and hence the position. Due to apodization and subsequent binarization, the radii of the circle blockers differ from those specified. In addition, when overlapped circles are formed, the edges of the circles that are in between two circles are not easily found; in that case, the input circle radius and location are chosen if the darkness of the location is within a tolerance. The overlapped internal circles are refined by matched filtering if more than half of the edges remain intact. Next, a verification step is carried out by the algorithm. If the new location is more than three pixels away from the specified position, then the darkness produced at the specified location is checked; if it satisfies a minimum darkness criterion, then the original location is accepted as the new location. Also the darkness at the new location is verified to be less than a certain threshold. 


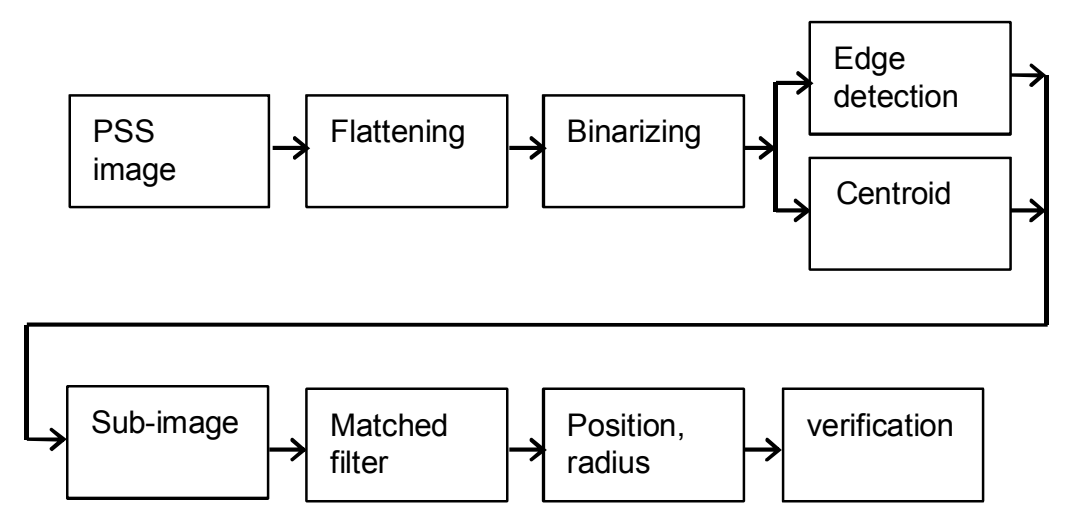

Figure 6. Block diagram of PSS calibration blocker detection algorithm.

\subsection{Detecting the line blocker}

The steps carried out in the line blocker detection are shown in Figure 7. The blocker image and the equation of the first line are input and used to rotate the image. A region of interest around the nominal bounding box is formed around the beam and the line is limited or cropped to this region. A bounding box is formed around the line using nominal values and a sub-image is created for processing.

The sub-image is divided into bands and in each band, the mean profile is calculated resulting in a profile vector or signal in the region of the band. The signal is then processed to find the optimal differential edge in the band. This process is repeated for all bands and yields a vector of points which approximate the edge of the blocker being verified.

Next a fit to the line is performed using a general Hough transform from the resulting points. The advantage of this method is that the line fit is rotationally invariant within the limits of the width of the band. This approach helps minimize the uncertainty associated with the apodized edges on the blockers under varying illumination conditions.

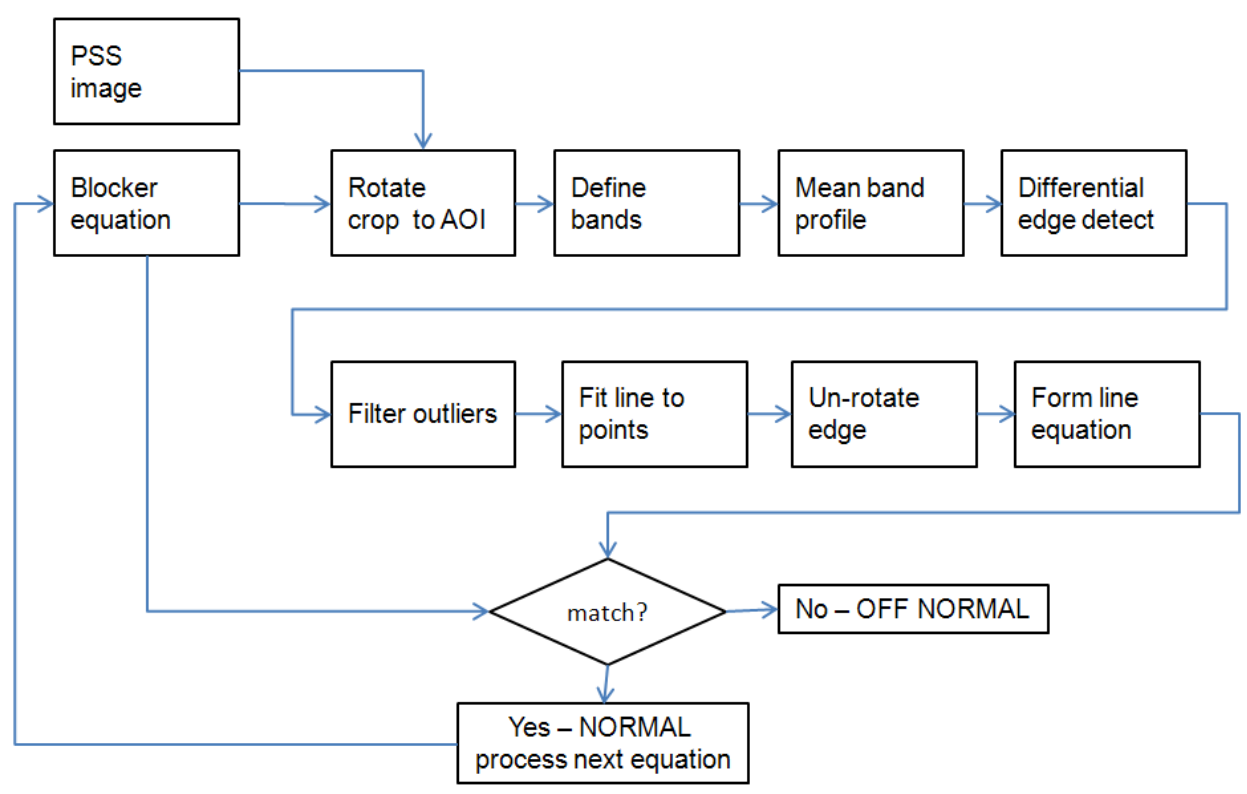

Figure 7. Block diagram of PSS line blocker detection algorithm. 
The line is then un-rotated to its original orientation and compared to the input line. A match within tolerance requirements outputs a normal condition, and the next blocker equation is processed. A line blocker that is out of tolerance results in an off-normal condition and is reported in the error log.

\section{CONCLUSIONS}

In this paper, the control system and underlying image processing required to deploy and verify various types of beam blockers are discussed. Real-time operation is facilitated by this database-driven, SLM-based system, which allows various types and sizes of beam blockers to be deployed.

\section{ACKNOWLEDGEMENTS}

This work performed under the auspices of the U.S. Department of Energy by Lawrence Livermore National Laboratory under Contract DE-AC52-07NA27344. The authors acknowledge valuable comments made by Steve Azevedo, Paul Van Arsdall and Paul Wegner on this paper.

\section{REFERENCES}

1. E. I. Moses, "Overview of the National Ignition Facility”, Fusion Science and Technology 54, pp. 361-366 (2008).

2. J. V. Candy, W. A. McClay, A. A. S. Awwal, and S. W. Ferguson, "Optimal position estimation for the automatic alignment of a high-energy laser," Journal of Optical Society of America A, Vol. 22, pp. 1348-1356, 2005.

3. R. A. Zacharias, N. R. Beer, E. S. Bliss, et al., "Alignment and wavefront control systems of the National Ignition Facility,” Optical Engineering 43, 2873-2884 (2004).

4. K. Wilhelmsen, A. Awwal, W. Ferguson, B Horowitz, V. Miller Kamm, C. Reynolds, "Automatic Alignment System For The National Ignition Facility", Proceedings of 2007 International Conference on Accelerator and Large Experimental Control Systems (ICALEPCS07), 486-490, Knoxville, Tennessee (2007). http://accelconf.web.cern.ch/accelconf/ica07/PAPERS/ROAA02.PDF

5. John Heebner, Michael Borden, Phil Miller, Christopher Stolz, Tayyab Suratwala, Paul Wegner, Mark Hermann, Mark Henesian, Chris Haynam, Steve Hunter, Kim Christensen, Nan Wong, Lynn Seppala, Gordon Brunton, Eddy Tse, Abdul Awwal, Mark Franks, Ed Marley, Kevin Williams, Michael Scanlan, Tracy Budge, Marcus Monticelli, Dan Walmer, Sham Dixit, Clay Widmayer, Justin Wolfe, Jeff Bude, Kelly McCarty, and Jean-Michel DiNicola, "A Programmable Beam Shaping System for Tailoring the Profile of High Fluence Laser Beams," Boulder Laser damage conference, 2010.

6. A. VanderLugt, "Signal Detection by Complex Spatial Filtering”, IEEE Trans. Inf. Theory IT-10, 139-145 (1964).

7. H. L. Van Trees, Detection, Estimation, and Modulation Theory, Part I (Wiley, 2001).

8. A. A. S. Awwal, Wilbert A. McClay, Walter S. Ferguson, James V. Candy, Thad Salmon, and Paul Wegner, "Detection and Tracking of the Back-Reflection of KDP Images in the presence or absence of a Phase mask," Applied Optics, Vol. 45, pp. 3038-3048, May 2006.

9. A. A. S. Awwal, Kenneth L. Rice, Tarek M. Taha, "Fast implementation of matched-filter-based automatic alignment image processing," Optics \& Laser Technology 41, 193-197 (2009).

10. M. A. Karim and A. A. S. Awwal, Optical Computing: An Introduction, John Wiley, New York, NY, 1992.

11. A. A. S. Awwal, "What can we learn from the shape of a correlation peak for position estimation?," Applied Optics, Vol. 49, pp. B41-B50, April 1, 2010. 